Canadian Mathematical Bulletin

doi:10.4153/CMB-2011-037-9

(C) Canadian Mathematical Society 2011

\title{
Extension of Some Theorems of W. Schwarz
}

\section{Michael Coons}

Abstract. In this paper, we prove that a non-zero power series $F(z) \in \mathbb{C}[[z]]$ satisfying

$$
F\left(z^{d}\right)=F(z)+\frac{A(z)}{B(z)},
$$

where $d \geq 2, A(z), B(z) \in \mathbb{C}[z]$ with $A(z) \neq 0$ and $\operatorname{deg} A(z), \operatorname{deg} B(z)<d$ is transcendental over $\mathbb{C}(z)$. Using this result and a theorem of Mahler's, we extend results of Golomb and Schwarz on transcendental values of certain power series. In particular, we prove that for all $k \geq 2$ the series $G_{k}(z):=\sum_{n=0}^{\infty} z^{k^{n}}\left(1-z^{k^{n}}\right)^{-1}$ is transcendental for all algebraic numbers $z$ with $|z|<1$. We give a similar result for $F_{k}(z):=\sum_{n=0}^{\infty} z^{k^{n}}\left(1+z^{k^{n}}\right)^{-1}$. These results were known to Mahler, though our proofs of the function transcendence are new and elementary; no linear algebra or differential calculus is used.

\section{Introduction}

Golomb proved in [4] that the values of the functions

$$
\sum_{n=0}^{\infty} \frac{z^{2^{n}}}{1+z^{2^{n}}} \quad \text { and } \quad \sum_{n=0}^{\infty} \frac{z^{2^{n}}}{1-z^{2^{n}}}
$$

are irrational at $z=\frac{1}{t}$ for $t=2,3,4, \ldots$, the interesting special case of which is that the sum of the reciprocals of the Fermat numbers is irrational. Schwarz [11] gave results on series of the form

$$
G_{k}(z):=\sum_{n=0}^{\infty} \frac{z^{k^{n}}}{1-z^{k^{n}}} .
$$

In particular, he proved that if $k, t$ and $b$ are integers satisfying $k \geq 2, t \geq 2$, and $1 \leq b<t^{1-1 / k}$, then the number

$$
G_{k}\left(b t^{-1}\right)=\sum_{n=0}^{\infty} \frac{b^{k^{n}}}{t^{k^{n}}-b^{k^{n}}}
$$

is irrational. Schwarz also showed that for $k, t, b \in \mathbb{N}$ with $k>2, t \geq 2$, and $1 \leq b<t^{1-5 / 2 k}$ the number $G_{k}\left(b t^{-1}\right)$ is transcendental. The case $k=2$ proved to

Received by the editors October 21, 2008; revised November 14, 2008.

Published electronically March 10, 2011.

AMS subject classification: 11B37, 11J81.

Keywords: functional equations, transcendence, power series. 
be more difficult, though he was able to show that for an integer $t \geq 2$, the number $G_{2}\left(t^{-1}\right)$ is not algebraic of the second degree.

Schwarz also remarked [11] that, "the irrationality of

$$
F_{k}\left(b t^{-1}\right):=\sum_{n=0}^{\infty} b^{k^{n}}\left(t^{k^{n}}+b^{k^{n}}\right)^{-1}
$$

for $k>2$ is unsettled" (here the notation $F_{k}\left(b t^{-1}\right)$ has been added).

Recently, Duverney [1] proved the transcendence of $G_{2}\left(t^{-1}\right)$ for integers $t \geq 2$ and extended Schwarz's transcendence results for the case $k=2$. He proved the following theorem.

Theorem 1.1 Let $a \geq 2$ be an integer and let $b_{n}$ be a sequence of integers satisfying $\left|b_{n}\right|=O\left(\eta^{-2^{n}}\right)$ for every $\eta \in(0,1)$. Suppose that $a^{2^{n}}+b_{n} \neq 0$ for every $n \in \mathbb{N}$. Then the number

$$
S=\sum_{n=0}^{\infty} \frac{1}{a^{2^{n}}+b_{n}}
$$

is transcendental.

We extend Schwarz's results further (to the best possible); in particular, we prove that for all $k \geq 2$ the series $G_{k}(z)=\sum_{n=0}^{\infty} z^{k^{n}}\left(1-z^{k^{n}}\right)^{-1}$ is transcendental for all algebraic numbers $z$ with $|z|<1$. We also prove the same result for $F_{k}(z)=$ $\sum_{n=0}^{\infty} z^{k^{n}}\left(1+z^{k^{n}}\right)^{-1}$ which settles the irrationality question of Schwarz's remark. These results were known to Mahler (see [5-8]), though our proofs of the function transcendence are new and elementary, coming from the proof of our main result; no linear algebra or differential calculus is used.

Our main result is that a non-zero power series $F(z) \in \mathbb{C}[[z]]$ satisfying

$$
F\left(z^{d}\right)=F(z)+\frac{A(z)}{B(z)}
$$

where $A(z), B(z) \in \mathbb{C}[z]$ with $A(z) \neq 0$ and $\operatorname{deg} A(z), \operatorname{deg} B(z)<d$ is transcendental over $\mathbb{C}(X)$. This extends a theorem of Nishioka [9] that states that $F(z)$ is either transcendental or rational.

\section{A General Theorem}

Nishioka [9] has shown the following.

Theorem 2.1 Suppose that $F(z) \in \mathbb{C}[[z]]$ satisfies one of the following for an integer $d>1$.

(i) $F\left(z^{d}\right)=\varphi(z, F(z))$,

(ii) $F(z)=\varphi\left(z, F\left(z^{d}\right)\right)$,

where $\varphi(z, u)$ is a rational function in $z$, u over $\mathbb{C}$. If $F(z)$ is algebraic over $\mathbb{C}(z)$, then $F(z) \in \mathbb{C}(z)$. 
Nishioka's proof of Theorem 2.1 relies heavily on deep ideas from algebraic number theory. In this section we provide an elementary proof of a special case of Theorem 2.1. In this special case, we are able to refine the conclusion by eliminating the possibility of $F(z)$ being a rational function.

Theorem 2.2 If $F(z)$ is a power series in $\mathbb{C}[[z]]$ satisfying

$$
F\left(z^{d}\right)=F(z)+\frac{A(z)}{B(z)}
$$

where $d \geq 2, A(z), B(z) \in \mathbb{C}[z]$ with $A(z) \neq 0$ and $\operatorname{deg} A(z), \operatorname{deg} B(z)<d$, then $F(z)$ is transcendental over $\mathbb{C}(z)$.

Proof Suppose that the power series $F(z)$ is algebraic and satisfies

$$
\sum_{r=0}^{n} q_{r}(z) F(z)^{r} \equiv 0,
$$

where the $q_{r}(z)$ are rational functions with $q_{n}(z)=1$ and $n \geq 1$ is chosen minimally.

Substituting $z^{d}$ into (2.1) and using the functional relation gives

$$
0 \equiv \sum_{r=0}^{n} q_{r}\left(z^{d}\right) F\left(z^{d}\right)^{r}=\sum_{r=0}^{n} q_{r}\left(z^{d}\right)\left(F(z)+\frac{A(z)}{B(z)}\right)^{r} .
$$

Without loss of generality, suppose $B(z)$ is monic. Multiplying by $B(z)^{n}$ to clear fractions as well as an application of the binomial theorem yields

$$
\begin{aligned}
& 0 \equiv \sum_{r=0}^{n} q_{r}\left(z^{d}\right) B(z)^{n-r}(B(z) F(z)+A(z))^{r}= \\
& \sum_{r=0}^{n} q_{r}\left(z^{d}\right) B(z)^{n-r} \sum_{j=0}^{r}\left(\begin{array}{l}
r \\
j
\end{array}\right) B(z)^{j} F(z)^{j} A(z)^{r-j} .
\end{aligned}
$$

Taking the difference between (2.2) and $B(z)^{n}$ times (2.1) gives

$$
\begin{aligned}
Q(z):=\sum_{r=0}^{n} q_{r}\left(z^{d}\right) B(z)^{n-r} \sum_{j=0}^{r}\left(\begin{array}{l}
r \\
j
\end{array}\right) B(z)^{j} F(z)^{j} A(z)^{r-j} & \\
& -B(z)^{n} \sum_{r=0}^{n} q_{r}(z) F(z)^{r} \equiv 0 .
\end{aligned}
$$

Note that we may also write $Q(z)=\sum_{m=0}^{n} h_{m}(z) F(z)^{m} \equiv 0$.

We determine $h_{n}(z)$. The only term in $Q(z)$ that can contribute to the coefficient of $F(z)^{n}$ is the $r=n$ term of the sum (2.3), which, recalling that $q_{n}(z)=1$, is

$$
\sum_{j=0}^{n}\left(\begin{array}{c}
n \\
j
\end{array}\right) B(z)^{j} F(z)^{j} A(z)^{n-j}-B(z)^{n} F(z)^{n},
$$


and only the $j=n$ term here contributes. Hence

$$
h_{n}(z)=\left(\begin{array}{l}
n \\
n
\end{array}\right) B(z)^{n} A(z)^{n-n}-B(z)^{n}=0
$$

so that $Q(z)=\sum_{m=0}^{n-1} h_{m}(z) F(z)^{m} \equiv 0$. Since $n$ was chosen minimally, $h_{m}(z) \equiv 0$ for all $m=0,1, \ldots, n-1$.

Using (2.3), we have that

$$
h_{m}(z)=\sum_{r=m}^{n}\left(\begin{array}{c}
r \\
m
\end{array}\right) q_{r}\left(z^{d}\right) B(z)^{n-r+m} A(z)^{r-m}-B(z)^{n} q_{m}(z) .
$$

Since $h_{n-1}(z) \equiv 0$, we have

$$
\sum_{r=n-1}^{n}\left(\begin{array}{c}
r \\
n-1
\end{array}\right) q_{r}\left(z^{d}\right) B(z)^{n-r+(n-1)} A(z)^{r-(n-1)}=B(z)^{n} q_{n-1}(z),
$$

so that removal of shared factors and again recalling $q_{n}(z)=1$, we have the identity

$$
q_{n-1}\left(z^{d}\right) B(z)+n A(z)=B(z) q_{n-1}(z) .
$$

Write $q_{n-1}(z)=\frac{\alpha(z)}{\beta(z)}$ where $\alpha(z), \beta(z) \in \mathbb{C}[z]$ with $\operatorname{gcd}(\alpha(z), \beta(z))=1$ and $\beta(z)$ monic. Then (2.4) becomes

$$
\beta(z) \alpha\left(z^{d}\right) B(z)+n \beta(z) \beta\left(z^{d}\right) A(z)=\beta\left(z^{d}\right) B(z) \alpha(z) .
$$

Equation (2.5) yields $\beta\left(z^{d}\right) \mid \beta(z) \alpha\left(z^{d}\right) B(z)$. As $\operatorname{gcd}\left(\alpha\left(z^{d}\right), \beta\left(z^{d}\right)\right)=1$, this implies that $\beta\left(z^{d}\right) \mid \beta(z) B(z)$. Therefore, $d \cdot \operatorname{deg} \beta(z) \leq \operatorname{deg} \beta(z)+\operatorname{deg} B(z)<\operatorname{deg} \beta(z)+d$. Hence

$$
0 \leq \operatorname{deg} \beta(z)<1+\frac{1}{d-1},
$$

so that since $d \geq 2$, either $\operatorname{deg} \beta(z)=0$ or $\operatorname{deg} \beta(z)=1$.

Suppose $\operatorname{deg} \beta(z)=0$ so that $\beta(z) \in \mathbb{C}$. Hence $\beta(z)=\beta\left(z^{d}\right) \in \mathbb{C}$; write $\beta:=$ $\beta(z)$. Now (2.5) becomes

$$
\alpha\left(z^{d}\right) B(z)+n \beta A(z)=B(z) \alpha(z) .
$$

Thus $B(z) \mid n \beta$, so that $\operatorname{deg} B(z)=0$; write $B:=B(z)$. So (2.6) becomes

$$
\alpha\left(z^{d}\right) B+n \beta A(z)=B \alpha(z),
$$

which implies that $d \cdot \operatorname{deg} \alpha(z)=\operatorname{deg} A(z)<d$, so that $\operatorname{deg} \alpha(z)=0$. Equation (2.7) and $\operatorname{deg} \alpha(z)=0$ imply that $A(z)=0$, which is impossible.

Now suppose $\operatorname{deg} \beta(z)=1$ and write $\beta(z)=z-\beta$. Comparing degrees in (2.5) implies that $\operatorname{deg} \alpha(z) \leq 1$. 
Recall that $\beta\left(z^{d}\right) \mid \beta(z) B(z)$ by (2.5). As $\operatorname{deg} B<d$, this implies that $\operatorname{deg} B=d-1$. Since $\beta$ and $B$ are both monic, we conclude that $\beta\left(z^{d}\right)=\beta(z) B(z)$, whence

$$
\frac{\beta\left(z^{d}\right)}{\beta(z)}=B(z) .
$$

Suppose that $\operatorname{deg} \alpha(z)=1$. Write $\alpha(z)=\delta(z-\alpha)$ and note that $\beta \neq \alpha$. In this case, replacing $B(z)$ in (2.5) and solving for $A(z)$ gives

$$
A(z)=\frac{\delta(\beta-\alpha) z\left(z^{d-1}-1\right)}{n(z-\beta)^{2}} \in \mathbb{C}[z] .
$$

Since $A(z) \in \mathbb{C}[z]$ we have that $(z-\beta)^{2} \mid\left(z^{d-1}-1\right)$, which is impossible because $z\left(z^{d-1}-1\right)$ has only simple roots; hence $\operatorname{deg} \alpha(z)=0$.

If $\operatorname{deg} \alpha(z)=0$, write $\alpha:=\alpha(z)$. Then writing $\beta(z)=z-\beta$ and solving (2.5) for $A(z)$, we have that

$$
A(z)=\frac{\alpha z\left(z^{d-1}-1\right)}{n(z-\beta)^{2}} \in \mathbb{C}[z],
$$

which is, again, impossible. Thus the theorem is proved.

\section{The Series $G_{k}(z)$ and $F_{k}(z)$}

To prove the transcendence results surrounding $G_{k}(z)$ and $F_{k}(z)$, we apply Theorem 2.2 as well as the following theorem of Mahler [5], as taken from Nishioka's book [10]. Here $\mathbf{I}$ is the set of algebraic integers over $(\mathbb{Q}, K$ is an algebraic number field, $\mathbf{I}_{K}=K \cap \mathbf{I}$, and $f(z) \in K[[z]]$ with radius of convergence $R>0$ satisfying the functional equation for an integer $d>1$,

$$
f\left(z^{d}\right)=\frac{\sum_{i=0}^{m} a_{i}(z) f(z)^{i}}{\sum_{i=0}^{m} b_{i}(z) f(z)^{i}}, \quad m<d, a_{i}(z), b_{i}(z) \in \mathbf{I}_{K}[z],
$$

and $\Delta(z):=\operatorname{Res}(A, B)$ is the resultant of

$$
A(u)=\sum_{i=0}^{m} a_{i}(z) u^{i} \quad \text { and } \quad B(u)=\sum_{i=0}^{m} b_{i}(z) u^{i}
$$

as polynomials in $u$.

Theorem 3.1 ([5]) Assume that $f(z)$ is not algebraic over $K(z)$. If $\alpha$ is an algebraic number with $0<|\alpha|<\min \{1, R\}$ and $\Delta\left(\alpha^{d^{n}}\right) \neq 0(n \geq 0)$, then $f(\alpha)$ is transcendental.

Now consider the functional equation $f\left(z^{k}\right)=f(z)-\frac{z}{1-z}$ with $k \geq 2$. Repeated use gives

$$
f\left(z^{k^{m}}\right)=f\left(z^{k^{m-1}}\right)-\frac{z^{k^{m-1}}}{1-z^{k^{m}}}=f(z)-\sum_{n=1}^{m} \frac{z^{k^{m-n}}}{1-z^{k^{m-n}}} .
$$


Changing the index and setting $W_{m}(z):=\sum_{n=0}^{m-1} z^{k^{n}} /\left(1-z^{k^{n}}\right)$ gives

$$
f(z)=f\left(z^{k^{m}}\right)+W_{m}(z) .
$$

In the region $|z|<1$ we have

$$
f(z)=\lim _{m \rightarrow \infty}\left[f\left(z^{k^{m}}\right)+W_{m}(z)\right]=\sum_{n=0}^{\infty} \frac{z^{k^{n}}}{1-z^{k^{n}}}=G_{k}(z) .
$$

This proves the following lemma.

Lemma 3.2 The function $G_{k}(z)$ satisfies the functional equation

$$
G_{k}\left(z^{k}\right)=G_{k}(z)+\frac{z}{z-1} .
$$

As a corollary of Theorem 2.2, we have the following corollary.

Corollary 3.3 The function $G_{k}(z)$ is transcendental over $\mathbb{C}(z)$.

To get the transcendence of the associated numbers, we use Mahler's theorem.

Proposition 3.4 For $k \geq 2$ and $z=\alpha$ algebraic with $0<|\alpha|<1, G_{k}(\alpha)$ is transcendental over $(\mathrm{O})$.

Proof Lemma 3.2 gives the functional equation

$$
G_{k}\left(z^{k}\right)=\frac{(1-z) G_{k}(z)-z}{1-z}
$$

so that, in the language of Theorem 3.1, we have $A(u)=(1-z) u-z$ and $B(u)=1-z$, $m=1<k=d$, and $a_{i}(z), b_{i}(z) \in \mathbf{I}_{K}[z]$. Since $B(u)$ is a constant polynomial in $u, \Delta(z):=\operatorname{Res}(A, B)=1-z$. Let $|\alpha|<1$ be algebraic; it is immediate that $\Delta\left(\alpha^{k^{n}}\right)=1-\alpha^{k^{n}} \neq 0 \quad(n \geq 0)$. Since $G_{k}(z)$ is not algebraic over $\mathbb{C}(z)$ (as supplied by Theorem 2.2), applying Theorem 3.1 we have that $G_{k}(\alpha)$ is transcendental over (O).

Corollary 3.5 If $k, b, t \in \mathbb{N}$ with $k \geq 2$ and $0<b<t$, then the number $G_{k}\left(b t^{-1}\right)$ is transcendental over $(\mathrm{O})$.

Proof Set $\alpha=b / t$ in Theorem 3.4 .

We turn now to the series

$$
F_{k}(z)=\sum_{n=0}^{\infty} \frac{z^{k^{n}}}{1+z^{k^{n}}} .
$$

Similar to $G_{k}(z), F_{k}(z)$ satisfies a functional equation,

$$
F_{k}\left(z^{k}\right)=F_{k}(z)-\frac{z}{z+1} .
$$

Using this functional equation, we have the following corollary of Theorem 2.2 . 
Corollary 3.6 The function $F_{k}(z)$ is transcendental over $\mathbb{C}(z)$.

As before, Mahler's theorem gives the following proposition.

Proposition 3.7 For $k \geq 2$ and $z=\alpha$ an algebraic number with $0<|\alpha|<1, F_{k}(\alpha)$ is transcendental over $(\mathrm{O})$.

Corollary 3.8 If $k, b, t \in \mathbb{N}$ with $k \geq 2$ and $1 \leq b<t$, then the number $F_{k}\left(b t^{-1}\right)$ is transcendental over $(\mathbf{O})$.

Remark 1 For some more recent work concerning results like Nishioka's Theorem 2.1, but for more general algebraic number fields, see [2] (This paper also contains a number of current references to work in this area). Also, concerning functions similar to $G_{k}(z)$ and $F_{k}(z)$ above, Duverney, Kanoko, and Tanaka [3] have given a complete classification of those series

$$
f(z):=\sum_{k=0}^{\infty} \frac{a^{k} z^{d^{k}}}{1+b z^{d^{k}}+c z^{2 d^{k}}} \in C[[z]]
$$

that are transcendental over $C(z)$ where $C$ is a field of characteristic $0, d \geq 2$, and $a, b, c \in C$ with $a \neq 0$.

Acknowledgments We wish to thank both Stephen Choi and the anonymous referee, whose comments and suggestions have helped clarify this exposition.

\section{References}

[1] D. Duverney, Transcendence of a fast converging series of rational numbers. Math. Proc. Cambridge Philos. Soc. 130(2001), no. 2, 193-207. doi:10.1017/S0305004100004783

[2] D. Duverney and K. Nishioka, An inductive method for proving the transcendence of certain series. Acta Arith. 110(2003), no. 4, 305-330. doi:10.4064/aa110-4-1

[3] D. Duverney, T. Kanoko, and T. Tanaka, Transcendence of certain reciprocal sums of linear recurrences. Monatsh. Math. 137 (2002), no. 2, 115-128. doi:10.1007/s00605-002-0501-4

[4] S. W. Golomb, On the sum of the reciprocals of the Fermat numbers and related irrationalities. Canad. J. Math. 15(1963), 475-478. doi:10.4153/CJM-1963-051-0

[5] K. Mahler, Arithmetische Eigenschaften der Lösungen einer Klasse von Funktionalgleichungen. Math. Ann. 101(1929), no. 1, 342-366. doi:10.1007/BF01454845

[6] Arithmetische Eigenschaften einer Klasse transzendental- transzendenter Funktionen. Math. Z. 32(1930), 545-585. doi:10.1007/BF01194652

[7] —Über das Verschwinden von Potenzreihen mehrerer Ver änderlicher in speziellen Punktfolgen. Math. Ann. 103(1930), no. 1, 573-587. doi:10.1007/BF01455711

[8] Lemarks on a paper by W. Schwarz. J. Number Theory 1(1969), 512-521. doi:10.1016/0022-314X(69)90013-4

[9] Keiji Nishioka, Algebraic function solutions of a certain class of functional equations. Arch. Math. 44(1985), no. 4, 330-335.

[10] Kumiko Nishioka, Mahler Functions and Transcendence. Lecture Notes in Mathematics, 1631, Springer-Verlag, Berlin, 1996.

[11] W. Schwarz, Remarks on the irrationality and transcendence of certain series. Math. Scand 20(1967), 269-274.

Department of Pure Mathematics, University of Waterloo, Waterloo, ON N2L 3G1

e-mail: mcoons@math.uwaterloo.ca 